\title{
Immersive Virtual Reality Anesthesiology Training: The Next Frontier in Simulation Training
}

\author{
Merrick $M$ Meese $M^{1^{*}}$, Zina Trost $P h D^{2}$ and Todd P Chang MD MAcM ${ }^{3}$ \\ ${ }^{1}$ Department of Anesthesiology \& Perioperative Medicine, The University of Alabama at Birmingham, USA \\ ${ }^{2}$ Department of Physical Medicine \& Rehabilitation, Virginia Commonwealth University, USA \\ ${ }^{3}$ Division of Emergency Medicine and Emergency and Transport Medicine, Children's Hospital Los Angeles, University of \\ Southern California, USA
}

Keywords

VR, Virtual reality, Stress

\section{Glossary of Terms}

VR: Virtual reality; CPR: Cardiopulmonary resuscitation; PTSD: Post-Traumatic Stress Disorder

\section{Introduction}

Current literature is unequivocal that simulation training and education are beneficial $[1,2]$. While anesthesia training for physicians and anesthetists - regularly features simulation as part of standard curricula, much of the currently available traditional simulation is focused on specific technical tasks abstracted from clinically realistic practice. Further, such training regularly calls for immense time and resources -- e.g., live presence of trained observers. There is thus limited opportunity for healthcare learners to engage in frequent repeatable simulations to refine care decision schemas within a safe environment that allows practice of critical decision-making and procedural skills with no patient risk. This is different from other industries that deal with high risk environments, such as aviation and the military that make extensive use of simulation to provide a safe environment for repetitive exposure and practice of decision making under stress. Where other industries practice extensively before deployment can have real-world ramifications, in healthcare, we often are only able to practice when it does. Can anyone imagine a professional sports player first attempting a new play on game day or the first time a new recruit deals with a jammed firearm is while in combat? Yet often the first time a medical trainee performs a new procedure or makes a clinical decision they have only read about, is when a real patient is involved. Healthcare is the only high-risk industry that does not routinely demand rehearsal prior to a high-risk endeavor. Even Broadway Theater is ahead of healthcare and anesthesiology in simulated practice.

Immersive virtual reality (VR) simulation is a currently emergent modality that has the potential to addresses the above limitations. The term immersive $V R$ is defined as a medium comprising interactive computer simulations that sense the participant's position and actions and replace or augment the feedback to one or more senses, giving the feeling of being mentally and physically immersed or present in the simulation [3]. The hardware utilized in immersive VR is usually some combination of a head-mounted display (providing visual and positional feedback/stimulus), headphones (auditory and positional feedback/stimulus) and controllers (input and positional feedback/stimulus). Compared to traditional screen-based and digital simulation [4], immersive VR provides a richer user experience, including a higher sense of presence and natural action. Immersive simulation approaches have already demonstrated positive results in other highrisk industries such as mining and spaceflight $[5,6]$.

\section{Reducing the time and resource burden of tradi- tional simulation}

As noted, traditional simulation can be expensive in terms of time and resources; manikin and standardized patient-based simulations require setup and preparation and providing

*Corresponding author: Merrick Meese, MD, Assistant Professor, Department of Anesthesiology \& Perioperative Medicine, UAB Medicine, The University of Alabama at Birmingham, JT 950, 619 South 19th Street, Birmingham, AL 35249-6810, USA, Tel: 205-996-7025

Accepted: March 22, 2020

Published online: March 24, 2020

Citation: Meese MM, Trost Z, Chang TP (2020) Immersive Virtual Reality Anesthesiology Training: The Next Frontier in Simulation Training. J Clin Anesth Pain Manag 4(1):97-100 


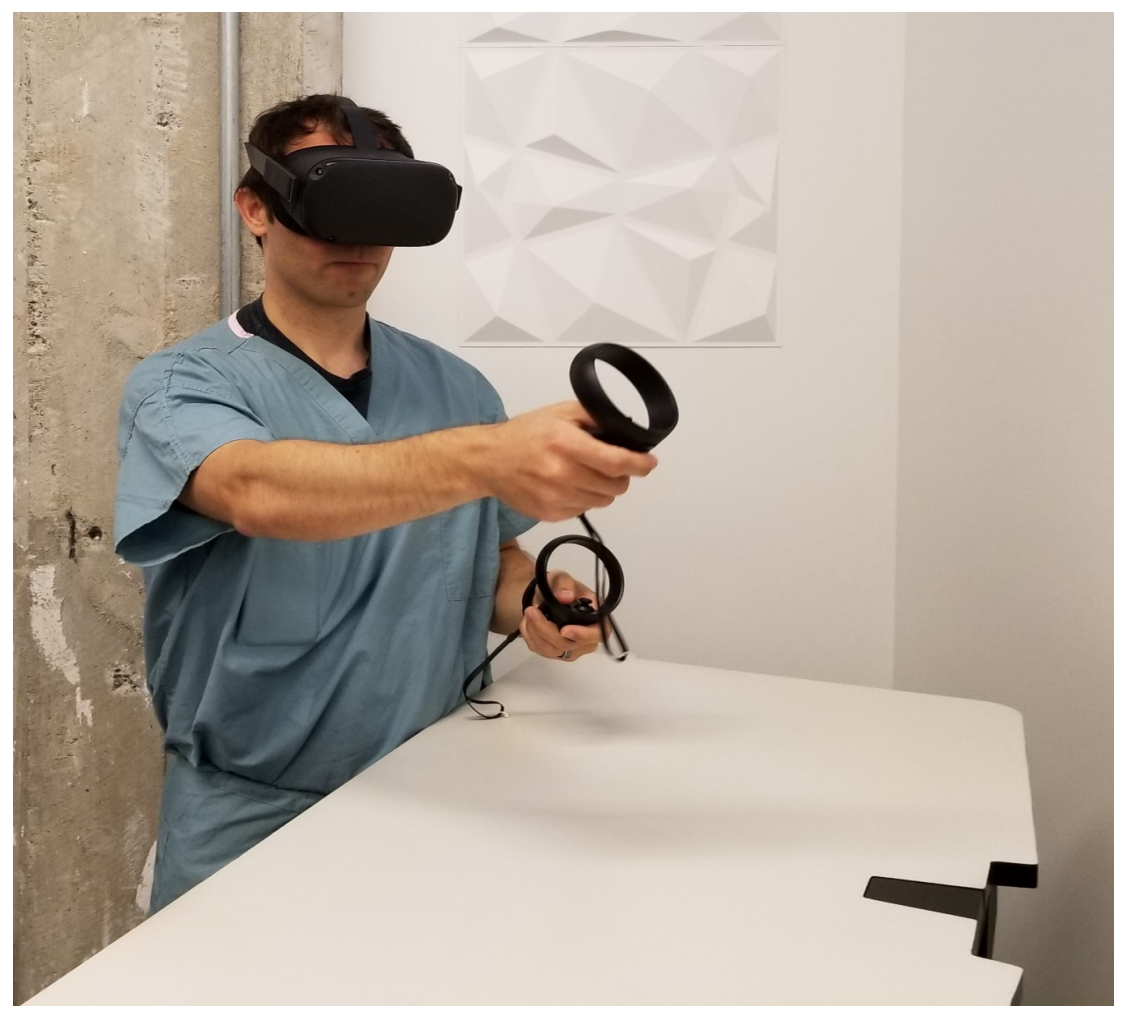

Figure 1: Demonstration of a learner using immersive virtual reality simulation hardware.

standard, identical experiences across groups and over time can be challenging. Furthermore, anesthesiology simulations require far more equipment and space than a simple bystander cardiopulmonary resuscitation (CPR) training. This includes simulated operating room space, an anesthesia machine, a large array of simulated medications, and a patient with particularly realistic or complex airways and physiologies. In contrast, immersive VR hardware is affordable and requires at maximum a $10^{\prime} \times 10^{\prime}$ area $(3 \mathrm{~m} \times 3 \mathrm{~m})$ or even smaller, with no physical set up. Newer hardware, such as the Oculus Quest, is very low cost and does not even require a traditional computer to function with very high fidelity and processing power (Figure 1). Thus, the "space" needed to conduct a simulation can be a small office or even in a user's home. Traditional simulation also regularly requires operations and logistics challenges, including arrival and time management, pre-simulation briefing, simulation preparation, performance, takedown, and debriefing. Immersive VR can collapse this complexity to a fraction of the time and does not need an extensive simulation center or staff.

Additionally, the fact that the simulation is entirely performed by a user on a standardized software package allows for a standard and consistent simulation experience, even more so than a trained, human confederate actor. This facet of the modality also allows for the ability to measure a user's task accomplishment, task omission, task sequencing, and task timeliness to generate a personalize report on areas needing improvement for the learner. This has significant ramifications on how future testing and evaluation of anesthesia providers could be conducted. Having a minimally demanding time and resource evaluation tool that could test a provider's context-sensitive understanding and dynamic response to time-sensitive and critical medical scenarios could be a major leap forward in how education and evaluation is conducted. This modality has the strong potential to allow for more accurate simulation evaluations across learners as they deal with clinical decision making and emergency situations.

Not only does reduced resource burden allow for increased simulation training opportunities for healthcare leaners within developed health systems, but this advantage opens the pathway to addressing a global lack of access to quality training. For example, a recent report by The Lancet found that five billion people are without access to safe surgical care and that in 2010 an estimate 16.9 million lives were lost from conditions needing safe surgical care [7]. To put this in perspective, that is more than the number of deaths caused by HIV/AIDS, tuberculosis and malaria combined. Increasing access to low resource, but high quality, simulation education has the ability to meet some of the demand for access to high quality training.

\section{Stress management for anesthesiologists}

High resource burden and low frequency are not the only issues that confront current simulation training. Current simulations rarely address provider or learner stress -a central factor in healthcare providers' personal and professional ability to provide care. Stress, in the clinical setting, is the sum total of external effects that negatively weigh down the clinician [8]. In many simulation studies, stress is manifested through physiology responses and through subjective feedback on excess anxiety, cognitive load, and/or distraction. While healthcare simulation ex- 
periences often focus on specific technical tasks or team communication goals, efforts to induce a stressful scenario are largely implicit. Simulation itself is meant to replicate a stressful scenario and research consistently demonstrates that simulation with high psychological fidelity can exacerbate stress physiology markers such as heart rate, heart rate variability, or cortisol levels [9]. However, very few simulations include the aim of stress experience and mitigation as a method of improving subsequent clinical performance. The need to address the role and impact of stress on providers is vital as provider burnout has reached the level of being a public healthcare crisis $[10,11]$. While "stress" encompasses multiple domains and its degree of impact on a person is multi-factorial, acute and high levels of stress have been clearly shown to negatively impact both technical and non-technical skills [12]. Several studies have demonstrated that a reduction in stress improves a medical provider's ability to perform tasks, and reduces cognitive load and burnout $[13,14]$. In addition to facilitating development of healthcare schemas, simulations of stressful and complex clinical scenarios allow learners to develop stress-management tools and reduce stress during actual clinical events [15]. In line with this, immersive VR has been shown to help with the management of Post-Traumatic Stress Disorder (PTSD) by carefully curating a stressful experience to process difficult emotional and cognitive reactions [16]. Because of the advantages of immersive VR simulation, the stressors experienced by learners can be precise and titrated. This functionality allows for the strong potential for an immersive and complex VR clinical scenario to encourage practicing stress management techniques such as stress exposure therapy, visualization, positive self-talk, etc. [17]. The ability to encounter and mitigate clinical stressors is imperative as we are reaching critical levels of provider burnout.

Yet another challenge of our current medical training is that learner exposure to rare and high-risk clinical scenarios is often unorganized, sporadic, and has inconsistent mentorship. The opportunity provided by an immersive VR simulation scenario is that rare, but critical, time-sensitive, high stress clinical scenarios can be repeatedly experienced by learners in high fidelity environment allowing for trial and error and optimization of response. Current literature strongly supports learner skill improvement and confidence with simulation use $[18,19]$. Anecdotally, one author has been in conversations with new staff physicians who have never taken care of a gunshot wound trauma during their residency due to the low prevalence of gun violence where they had their training. Having a readily available immersive simulation environment to supplement regionally specific training deficits would be a boon to training programs. Non-VR simulation is currently used to replace specific clinical experience in nursing education, particularly for rare events, and we should be using immersive VR simulation in a similar fashion to recreate particularly rare scenarios that could expose otherwise hidden clinical deficits among both resident and practicing anesthesiologists [20]. VR has the potential to mitigate specific deficits in exposure to high risk clinical scenarios by allowing for healthcare learners to repeatedly experience them in simulation.

\section{Current state of immersive virtual reality simu- lation}

Even non-immersive VR training is currently at the stage of early adoption. Yet, simulation educators are beginning to realize the advantages that VR training offers. Several high-fidelity simulators, such as Anesthesia SimSTAT (a screen based training program put out by the American Society of Anesthesiologists) and Simbionix's GI-Bronch Mentor Virtual Reality Simulator, are currently in wide use in anesthesiology training programs. That being said, there are a few companies that are developing immersive VR simulation training, companies such as CAE healthcare, SimX, and Oxford Medical Simulation. They offer products that are being used at academic hospitals in both Europe and the United States of America. Some examples of these scenarios include transport resuscitation, casualty care, and other medical emergencies. However, widespread adoption of immersive VR training is still limited to a few large academic centers, favoring medical schools over residency programs. This is due to the greater value proposition over a larger student body, as well as the current state of medical VR simulations in which checklists are often used at the level of novice or beginner, rather than complex skills at the expert level.

Surgical VR focuses on the psychomotor aspects of tissue or bone manipulation, from companies such as OssoVR, BioFlightVR, and PrecisionOS. Surgical VR is primarily concerned with the economy of motion and hand coordination skills, providing positional haptic feedback to determine correct placement of instruments, incisions, and implantations. Surgical VR does not emphasize the immersive situational awareness required for medical training, such as for anesthesiology. Situational awareness would include consideration of subtle vital signs, noticing patient changes, and reacting conceptually or with team communication. In short, Surgical VR takes advantage of the positional haptic feedback for which VR hand controllers or hand recognition excels; medical VR harnesses the total immersion capability of VR for situational awareness and clinical decision making.

There are still several challenges that must be faced when implementing immersive VR simulation training. Cybersickness is a limiting factor for some people, but judicious purchasing decisions and sufficient computing power with technological advances continue to minimize these effects. Another major challenge is in determine the types of learning and training for which immersive VR training is best suited. Many companies that are developing immersive VR are marketing their scenarios as replacements for current simulation modalities. The authors believe that, in most cases, this is likely not the best use for immersive VR, and that a complementary approach is most effective. Further research should determine the best use for this technology.

\section{Next steps}

Immersive VR has the potential to radically alter how simulation education and training is conducted. Just as each 
new educational modality simply augments the prior ones, including textbooks, paper journal articles, slide sets and Powerpoints ${ }^{\circledR}$, immersive VR will be a powerful adjuvant to traditional manikin and standardized patient-based simulations. Traditional simulation still allows for actual tactile performance of tasks, video recorded and human observational comments, instructor led de-briefs and interactions with other actors and team-members which are important for the simulation experience. This being acknowledged, immersive VR fills in the gap between full simulation center simulations and actual clinical care. This is particularly adept for practitioners in smaller or rural areas in which access to a large simulation center is difficult or costly. It minimizes time and resource burden, allowing for simulation education that fits into a learner's schedule. In much the same way a learner would open a text book for a refresher on a clinical situation they are unfamiliar with, they can easily load into an immersive VR simulation and practice clinical decision making in a dynamic and real time environment.

Despite having potentially profound benefits for simulation training and education, immersive VR simulation is still a nascent field. Screen based and digital learning only became widespread in the 1990s and immersive VR is a much younger - but exponentially evolving -- technology. In the recent years, there has been a growing body of work supporting the role of immersive VR within technical and clinical decision-making simulation. Yet, there is still a paucity of literature, data, and real-world directives on how to best utilize this new technology. We encourage the readers to take a look at how this tool can best be employed to improve medical training for both new learners as well as for continuing medical education. We believe that immersive VR is the next frontier in simulation with significant positive implications for simulation training.

\section{Financial Disclosures}

Dr. Meese currently has funding from grants awarded from the University of Alabama at Birmingham Health Services Foundation Graduate Education Fund of $\$ 99,196$, Faculty Development Grant of $\$ 10,000$ and the College of Arts and Sciences Interdisciplinary Team Award of $\$ 30,000$. Dr. Trost currently has funding from grants awarded from the University of Alabama at Birmingham Health Services Foundation Graduate Education Fund of $\$ 99,196$ and the College of Arts and Sciences Interdisciplinary Team Award of $\$ 30,000$. Dr. Chang has no disclosures.

\section{Conflicts of Interest}

Dr. Chang is the medical consultant to SBC Med Sim LLC, A.i.Solve, Ltd., and BreakAway Games, Ltd., but without any stock, salary support, or board membership position. Drs. Meese and Trost have no conflicts.

Merrick Meese: This author generated the idea for this paper as well as help with formatting and editing.

Zina Trost: This author helped with formatting, editing and idea consolidation.

Todd Chang: This author helped with formatting, editing and idea consolidation.

\section{References}

1. Cook DA, Hatala R, Brydges R, et al. (2011) Technology-enhanced simulation for health professions education: A systematic review and meta-analysis. JAMA 306: 978-988.

2. Zendejas B, Brydges R, Wang AT, et al. (2013) Patient outcomes in simulation-based medical education: A systematic review. J Gen Intern Med 28: 1078-1089.

3. Sherman WR, Craig AB (2018) Understanding virtual reality: Interface, application, and design. Morgan Kaufmann.

4. North MM, North SM (2016) A comparative study of sense of presence of traditional virtual reality and immersive environments. Australasian Journal of Information Systems 20.

5. Tichon D, Burgess-Limerick (2011) A review of virtual reality as a medium for safety related training in mining. 3: 33-40.

6. Finseth $T$, Keren N, Dorneich M, et al. (2018) Evaluating the effectiveness of graduated stress exposure in virtual spaceflight hazard training. Journal of Cognitive Engineering and Decision Making 12.

7. Meara JG, Leather AJM, Hagander L, et al. (2015) Global surgery 2030: Evidence and solutions for achieving health, welfare, and economic development. Lancet 386: 569-624.

8. Sood A, Prasad K, Schroeder D, et al. (2011) Stress management and resilience training among department of medicine faculty: A pilot randomized clinical trial. J Gen Intern Med 26: 858-861.

9. Chang TP, Beshay Y, Hollinger T, et al. (2019) Comparisons of stress physiology of providers in real-life resuscitations and virtual reality-simulated resuscitations. Simul Healthc 14: 104-112.

10. Jha A, llif A, Chaoui A (2019) A crisis in health care: A call to action on physician burnout. Massachusetts Medical Society.

11. Williams ES, Manwell LB, Konrad TR, et al. (2007) The relationship of organizational culture, stress, satisfaction, and burnout with physician-reported error and suboptimal patient care: results from the MEMO study. Health Care Manage Rev 32: 203212.

12. Arora S, Sevdalis N, Nestel D, et al. (2010) The impact of stress on surgical performance: A systematic review of the literature. Surgery 147: 318-330.

13. Boet S, Bould MD, Fung L, et al. (2014) Transfer of learning and patient outcome in simulated crisis resource management: $A$ systematic review. Can J Anaesth 61: 571-582.

14. Andreatta PB, Maslowski E, Petty S, et al. (2010) Virtual reality triage training provides a viable solution for disaster-preparedness. Acad Emerg Med 17: 870-876.

15. Riem N, Boet S, Bould MD, et al. (2012) Do technical skills correlate with non-technical skills in crisis resource management: $A$ simulation study. Br J Anaesth 109: 723-728.

16. Rizzo A, Reger G, Perlman K, et al. (2008) Virtual reality post traumatic stress disorder (PTSD) exposure therapy results with active duty Iraq war combatants.

17. Ignacio J, Dolmans D, Scherpbier A, et al. (2016) Stress and anxiety management strategies in health professions' simulation training: A review of the literature. BMJ Simulation \& Technology Enhanced Learning 2: 42-46.

18. Saleta JM (2012) The effects of utilizing high-fidelity simulation in medical residency programs.

19. Seemann NM (2016) The complex experience of surgeon stress in the operating room.

20. Curl ED, Smith S, Chisholm LA, et al. (2016) Effectiveness of integrated simulation and clinical experiences compared to traditional clinical experiences for nursing students. Nurs Educ Perspect 37: 72-77. 